\title{
Muerte e identidad femenina en el mundo argárico
}

\author{
Death and female identity in the Argaric world
}

Sandra Montón-Subías (*)

\section{RESUMEN}

Ya que la identidad se construye en un contexto de interacción social, es de suponer que el comportamiento funerario formaría parte de la construcción social de la identidad en las comunidades prehistóricas. Por lo tanto, también debió actuar como componente activo en la representación y negociación de las identidades sociales argáricas, incluida la femenina, cuyo estudio se propone este artículo.

En las siguientes páginas repasaré las prácticas funerarias argáricas, postularé nuevas hipótesis respecto a su realización y, destacaré, a través del estudio de la cultura material funeraria y de los cuerpos del pasado, de qué modo la identidad hegemónica de las mujeres argáricas se configuró a partir de mecanismos relacionales.

\begin{abstract}
Given that selves are constructed in contexts of social interaction, we can safely assume that funerary behaviour fostered the formation of subjective identity in prehistoric societies. Funerary behaviour can therefore be seen as an active component in representing and negotiating Argaric social identities, including female selves, which are the main focus of this essay.

An overview of Argaric funerary practices will be presented here, alongside new hypotheses on how they were conducted. A study of corpses and funerary material culture will show how it was predominantly through relational mechanisms that Argaric women's selves were constructed.
\end{abstract}

Palabras clave: Edad del Bronce; El Argar; Sudeste de la Península Ibérica; II milenio AC; Registro Funerario; Construcción de la identidad.

(*) Profesora de Investigación ICREA. Departamento de Humanidades, Universitat Pompeu Fabra. C/ Ramon Trias Fargas 25-27. Barcelona 08005.

Correo electrónico: sandra.monton@upf.edu

Recibido: 23-VII-2009; aceptado: 11-XI-2009.
Key words: Bronze Age; El Argar; Southeast of the Iberian Peninsula; Second millennium BC; Mortuary Record; Identity Formation.

\section{INTRODUCCIÓN}

En este artículo estudiaré la pauta funeraria de la cultura argárica en tanto que componente activo en la representación y construcción social de la identidad femenina en sus comunidades. Ya que la identidad se construye en un contexto de interacción social, se puede asumir que el comportamiento funerario formó parte de esa construcción en las comunidades prehistóricas y, por lo tanto, también en las argáricas (Fig. 1). Mediante su particular ritual funerario argáricos y argáricas expresaron y re-crearon a la vez distancias y proximidades sociales. Desde mi punto de vista, tan importantes resultan las vinculaciones como las diferencias que se articularon a través del espectro funerario, pues todas ellas resultaron de acciones humanas que

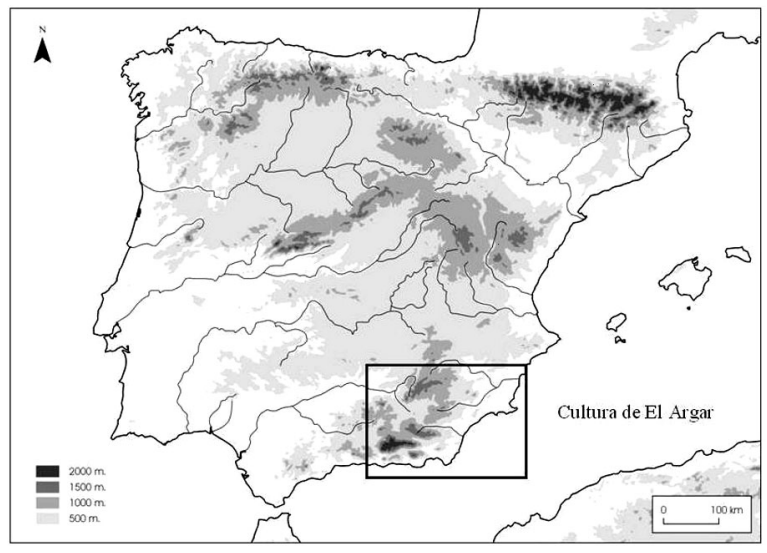

Fig. 1. Localización geográfica de la cultura de El Argar en el Sudeste de la Península Ibérica. 
pueden ofrecer información sobre la construcción social de la identidad.

Es de sobra conocida la importancia que el estudio del mundo de la muerte ha tenido desde sus orígenes para la arqueología. Las necrópolis y las costumbres funerarias han jugado un papel decisivo en la definición y caracterización de los grupos sociales del pasado. A partir de las estructuras, lugares y objetos que se eligieron al enterrar a los muertos se han formulado modelos interpretativos que, desde diferentes perspectivas, en ocasiones antagónicas, han intentando entender cómo era el mundo de esos muertos en vida, cómo se organizaba la comunidad en la que vivían, qué decidieron hacer con ellos quiénes permanecieron vivos y por qué (Childe 1945; Binford 1971; Brown 1971; Randsborg 1974; Shennan 1975; Peebles y Kus 1977; Chapman et al. 1981; Shanks y Tilley 1982; Lull y Picazo 1989; Parker Pearson 1999; Tarlow 1999; Lull 2000; Arnold y Wicker 2001, entre otros).

Los estudios sobre el mundo funerario se han desarrollado bajo la premisa de que los enterramientos y su contenido guardaron una relación estrecha con el papel social que tuvieron en vida las personas enterradas. Asumiendo la cautela apuntada por diferentes investigaciones, pienso que el contexto mortuorio no tiene por qué entenderse como reflejo mecánico de la vida en las sociedades prehistóricas (Parker Pearson 1999; Rautman y Talalay 2000; Sofaer 2006). Sin embargo, creo también que muerte y vida no son dos aspectos disociados y que, al menos en muchas sociedades etnográficas y del pasado, la muerte forma parte de ese continuum que representa el ciclo vital. De hecho, si a partir del análisis del registro funerario es posible entender aspectos de la vida de estas comunidades es porque el comportamiento funerario formó parte precisamente de sus interacciones vitales. Por ello, estudiar arqueológicamente el mundo de la muerte nos ayuda a entender la vida en las sociedades del pasado y nos aproxima a sus biografías.

Además, el registro funerario se define por su carácter deliberado y planificado frente a las pautas de deposición de otros contextos arqueológicos. Este hecho implica que, en términos generales, los depósitos funerarios sean cuidadosamente seleccionados y organizados conforme a premisas sociales predeterminadas que, aunque no necesariamente ofrecen una imagen completa o general de la sociedad analizada, precisamente por ese carácter deliberado, contienen mensajes directos e intencionados de la sociedad que los creó.

En los rituales funerarios se imbricaron aspectos de diferente índole, entre otros, normativas sociales, emociones personales, cultura material, percepciones sociales, posiciones y roles sociales de los difuntos, actividades que llevarían a cabo en vida o incluso el propio momento, esperado o no, de la muerte (Damm 1991; Chesson 2001; Joyce 2001).

Esta combinación de ingredientes debió operar también en las sociedades argáricas y en la configuración del ritual funerario que se conoce hoy en día. Por ello confío en que, si se ahonda en el estudio de dicho ritual, se comprenderá mejor quiénes eran los argáricos y argáricas que poblaron el Sudeste de la Península Ibérica desde c. 2250 hasta c. 1450 cal AC.

Como he anunciado, pretendo profundizar en la comprensión de los mecanismos mediante los cuales se configuró la identidad femenina en esas comunidades recurriendo básicamente a dos tipos de evidencia: el análisis de los ajuares funerarios (principalmente punzones) asociados a los cuerpos femeninos y la información paleoantropológica procedente de una muestra de esqueletos argáricos. Para asegurar la fiabilidad de los resultados cuantitativos he utilizado la evidencia procedente de necrópolis excavadas sistemáticamente con métodos modernos y de tumbas no expoliadas. Sólo en estos casos se puede contar con testimonios fiables sobre los diferentes aspectos que definen el comportamiento funerario. Soy consciente de que he excluido un buen número de las necrópolis argáricas clásicas, aunque he utilizado su información a nivel cualitativo. En este sentido, recientes trabajos demuestran los problemas e incertidumbres existentes a la hora de definir las adscripciones y asociaciones de elementos materiales con sepulturas concretas (Brandherm 2000; Andúgar 2006). Especialmente, la información procedente de la colección Siret, marcada por su disgregación y fuertemente condicionada por los avatares del siglo $\mathrm{XX}$, ha provocado no pocas dudas sobre las asociaciones contextuales en necrópolis como El Argar, El Oficio, Fuente Álamo o Herrerías. Estas incertidumbres me han conducido a manejar sólo aquellos datos que poseen unas mínimas garantías: 19 necrópolis y 167 tumbas no expoliadas.

Por último, las dataciones absolutas actualmente existentes, a pesar de los destacados es- 
fuerzos para contextualizar cronológicamente la materialidad funeraria argárica (Castro et al. 1993-94; Aranda et al. 2008), resultan insuficientes tanto por su escaso número como por su desigual distribución territorial. En un análisis general de las sociedades argáricas, como el que se pretende en este trabajo, no queda más remedio que arriesgarse a considerar sus pautas rituales como un todo. Por el momento, el desarrollo temporal de los elementos materiales asociados al ritual debe entenderse como tendencias generales que tendrán que ser convenientemente contrastadas con series radiométricas más amplias que afecten a las diferentes regiones de la geografía argárica. No obstante, la fuerte normalización del ritual observada en las sociedades argáricas asegura la suficiente unidad como para que trabajos de esta escala sean relevantes, independientemente de futuras matizaciones temporales.

\section{EL RITUAL FUNERARIO ARGÁRICO}

Desde que los hermanos Siret dieran nombre a la cultura de El Argar a finales del siglo XIX, la norma cultural argárica se definió conjugando tres aspectos considerados novedosos en relación al período anterior: el patrón de asentamiento, el ritual funerario y determinados elementos de cultura material. Los avances de la investigación de campo han exigido matizar esa pretendida novedad y unicidad. Tal vez el caso más evidente sea el referido al patrón de asentamiento, que ya no incluye únicamente la construcción de poblados en cerros o elevaciones estratégicamente situados, sino una variedad más amplia de asentamientos con características diferenciadas (Ayala 1991; Castro et al. 2001; Aranda et al. 2009b). Al haberse diversificado lo que antes se concebía como un patrón de asentamiento unitario, se ha agudizado la cualidad del ritual funerario (y de su característica cultura material) para servir como elemento clave en la definición de lo argárico, hecho que se acentúa, si cabe, en las áreas situadas en sus límites geográficos de distribución (Jover y López Padilla 1995, 1997; López Padilla et al. 2006).

Hace aproximadamente 4200-4000 años, los grupos argáricos del Sudeste de la Península Ibérica comenzaron a enterrar de una manera bastante diferente a la que hasta entonces debía de haberse considerado como tradicional. En vez de utilizar cementerios fuera de los poblados y estructuras de inhumación colectiva, las tumbas argáricas, destinadas por lo general a una o dos personas (aunque también se conocen enterramientos triples y cuádruples), empezaron a situarse dentro de los poblados, generalmente debajo de las viviendas. El espacio concebido como poblado pasó a albergar a muertos y vivos, de manera que muerte y vida formarían parte del paisaje de la cotidianeidad (Montón-Subías 2007). Esta nueva costumbre, que se mantuvo durante toda la cronología argárica, se fue generalizando hasta ocupar un territorio que actualmente comprende las provincias de Almería y Murcia, gran parte de Granada y Jaén, y determinadas comarcas de Alicante.

Desde sus inicios, buena parte de la investigación argárica ha pivotado sobre el estudio de estas prácticas funerarias y sobre todo de los contenedores y ajuares funerarios. A partir de su análisis se formuló, en un primer momento, la secuencia tipológico-cronológica tradicional (Siret y Siret 1890; Cuadrado 1950; Blance 1964, 1971; Schubart 1975) y más tarde, utilizando nuevos planteamientos teóricos, su organización social (Lull 1983; Lull y Estévez 1986; Contreras et al. 1987-88). Y si bien la secuencia cronológica ha sido contestada por las fechas de radiocarbono, y su aceptación ya no es unánime, sigue existiendo un elevado consenso sobre la naturaleza estratificada de la sociedad argárica (Gilman 1981, 2001) e, incluso, sobre su carácter estatal o para-estatal (Contreras et al. 1987-88; Arteaga 1992; Lull y Risch 1996; Jover y López Padilla 1997; Contreras 2000; Lull 2000; Castro et al. 2001; Cámara 2001; Chapman 2003; Aranda y Molina 2006).

Reconozco la aportación de los anteriores estudios para visualizar el comportamiento funerario de un modo más diáfano al que de otro modo sería posible, aunque pienso que el estado actual de la investigación permite emprender ya el tipo de análisis que se plantea en este trabajo. Los avances generados en el campo de los estudios sobre identidad en el pasado (Meskell 2001; Hernando 2002; Fowler 2004; Díaz-Andreu et al. 2005; Insoll 2007) posibilitan y exigen nuevas líneas que consideren que las decisiones que la gente del pasado adoptó en relación a dónde, cómo y con qué objetos enterrar a sus muertos guardaron una estrecha relación con las identidades sociales, y no sólo las de clase, que operaron en sus comunidades (Montón-Subías 2007; Sánchez-Romero 2008a; Aranda et al. 2009b). 


\section{2a. Los enterramientos argáricos}

Aunque existieron diferencias en la manera efectiva de implementar los enterramientos, posiblemente debidas a la propia idiosincrasia de las comunidades o de las personas que fueron enterradas, sin embargo creo que el hecho en sí del enterramiento, los contenedores que se utilizaron, los ajuares que se depositaron (cuando los hubo) y algunos de los ritos asociados pueden definir lo que podría calificarse de coiné funeraria argárica. A diferencia de lo que se ha planteado en otros trabajos (Lull y Estévez 1986), pienso que una de las principales vinculaciones a la comunidad argárica probablemente vino marcada por el derecho al enterramiento. El número sorprendentemente bajo de tumbas halladas en relación a las diferentes estimaciones demográficas sobre el número de habitantes de los poblados argáricos apunta a que no todo el mundo debió enterrarse (Chapman 1990, 2005). Por ello parece factible pensar que el acceso al enterramiento funcionara como principio de inclusión, como instrumento conectivo que, en este caso a partir de la muerte, identificara la pertenencia a la comunidad argárica, independientemente de otras posibles circunstancias sociales.

Uno de los aspectos esenciales del ritual sería la propia exposición del cadáver. Poco se conoce sobre las prácticas específicas al respecto, pero por la posición flexionada que adoptaron los cuerpos en el interior de las tumbas, se sabe que entre el momento de la muerte y su introducción en la tumba pasó el tiempo suficiente para que el rigor mortis, que normalmente dura unas 48 horas, no impidiese flexionar el cadáver. La aparición en Galera de dos cuerpos parcialmente momificados nos aproxima al aspecto que debieron tener durante este período (Molina et al. 2003). También se han documentado fragmentos de tejido en varias tumbas (Alfaro 1984) y restos de ocre en algunos huesos, sobre todo en cráneos, que, según creyeron ya los Siret (1890), corresponderían a trazas dejadas por telas pintadas con cinabrio (Delibes 2000).

Transcurrido el tiempo necesario, los cuerpos se depositaban en el interior de las tumbas: urnas cerámicas, cistas, fosas o covachas. En el estudio estadístico de los tipos de sepulturas se advierte una clara tendencia a la elección del binomio fosa-cista en las tierras argáricas del interior, mientras que en las comarcas del Sudeste en sen- tido estricto dominaría la elección cista-urna (Lull y Estévez 1986).

En la mayoría de los casos parece que existe una normalización fuerte en la construcción de las tumbas, pero se constatan también excepciones notorias que sin duda merecen una explicación. Pondré solo dos ejemplos: las cistas de tamaño excepcional del yacimiento de El Rincón de Almendricos (García del Toro y Ayala 1978) y las grandes fosas abiertas en la roca, como la sepultura 21 del Cerro de la Encina (Aranda et al. 2008).

Generalmente se ha contrapuesto el enterramiento individual argárico al colectivo calcolítico, aunque no siempre en El Argar las inhumaciones fueron individuales. En el Cerro de la Encina, las sepulturas con más de una inhumación suponen el $68 \%$ del total, lo que contrasta fuertemente con la situación en otras necrópolis argáricas donde la inhumación individual es absolutamente mayoritaria (Aranda y Molina 2006; Aranda et al. 2008). En cualquiera de las zonas argáricas pueden encontrarse ambos tipos de enterramiento, aunque, efectivamente, en comarcas como la vega de Granada el número de sepulturas dobles, triples e incluso cuádruples supone una particularidad.

La mayoría de los enterramientos no individuales no fueron sincrónicos sino que las tumbas se reabrieron con posterioridad para albergar otros cuerpos desplazando y desarticulando los restos óseos de la/s inhumación/es previa/s. Según algunos autores, estos intervalos temporales podrían diferir incluso en tres generaciones respecto al primer inhumado (Castro et al. 1993-94). La tendencia al enterramiento femenino con anterioridad al masculino, la distancia temporal existente entre ambos y la mayor movilidad masculina son los argumentos utilizados por estos mismos investigadores para proponer un modelo de parentesco basado en la matrilocalidad y la matrilinealidad. No obstante, la escasez de dataciones en las que se sustenta esta propuesta junto a los datos procedentes de otras necrópolis, indican que todavía debe mostrarse cautela por lo que se refiere al margen exacto de la distancia temporal. Por ejemplo, en la sepultura 21 del Cerro de la Encina se documenta un enterramiento doble de hombre y mujer en perfecta conexión anatómica y una clara superposición entre ambos (Aranda et al. 2008). El primero en ser depositado fue el hombre. Sus pies y parte de sus tibias y peronés 
aparecen bajo el cuerpo de la mujer. Frente a la certidumbre de la superposición estratigráfica de ambas inhumaciones, las dataciones ofrecen una visión opuesta, pues la fecha del hombre es más reciente que la de la mujer. La primera se sitúa en el 1555 cal. BC con un intervalo de calibración a $1 \sigma$ de 1610-1500 cal. BC, frente a la datación de la mujer de $1600 \mathrm{cal}$. BC con un intervalo a $1 \sigma$ de 1670-1530 cal. BC. Esta aparente contradicción aconseja una mayor prudencia en la consideración de la distancia temporal en la deposición de inhumaciones dobles o triples, sobre todo cuando existe un solapamiento en el intervalo de $1 \sigma$ que, como se sabe, supone una probabilidad del $68 \%$. En el caso de la sepultura 21 el intervalo de solapamiento sería de 1610-1530 cal. BC, momento en que muy probablemente se produjo el enterramiento de ambos individuos. La diferencia entre ambas dataciones solo se explica si se tiene en cuenta los intervalos de calibración: a $1 \sigma$ la coincidencia es del $47 \%$ y a $2 \sigma$, en donde la probabilidad aumenta al $95 \%$, el solapamiento entre ambas fechas es del $60 \%$. La superposición estratigráfica de ambos individuos en la sepultura 21 y su cronología demuestran que la valoración de la distancia temporal en sepulturas dobles de hombre-mujer requiere de amplias series de dataciones en donde los intervalos de calibración puedan ser valorados estadísticamente (Aranda et al. 2008). Algo parecido ocurre en la sepultura TI de la Illeta dels Banyets, donde la datación de la mujer, que es la que no presenta conexión anatómica, es más reciente que la del hombre (López et al. 2006). Por lo tanto, parece que el debate en torno al modelo de parentesco argárico continúa abierto y que las variables geográficas y temporales también pueden haber tenido un peso relevante.

Como también he apuntado ya, durante el período argárico generalmente se enterraba bajo los pisos de las casas, de modo que estas acogían a un tiempo a muertos y a vivos en un mismo espacio. Probablemente esta práctica expresaba la voluntad de marcar simbólicamente la continuidad del ciclo de la vida en estas sociedades y denotar que los difuntos formaban parte de la vida cotidiana del poblado. Asimismo, también a este respecto existen excepciones, tanto en asentamientos limítrofes como de las zonas nucleares, al haberse constatado sepulturas fuera del área de los poblados.

Tras depositar al cadáver junto con el ajuar elegido dentro de la tumba, aspecto que trataré en la siguiente sección, se procedería a su cierre. En algunos yacimientos se aduce la existencia de pequeñas agrupaciones de piedra encima de las tumbas a modo de señalización (Martínez Rodríguez et al. 1996). En cualquier caso, parece evidente que debía conocerse dónde se localizaba la tumba ya que muchas de ellas se reutilizaron.

No incluyo en los análisis los llamados cenotafios, que aparecen principalmente en yacimientos de Murcia y Almería. Recientemente se han constatado en El Rincón, Cerro de las Viñas, Lorca -Calle Zapatería-, la Loma del Tío Ginés y Gatas, pero ya se sabía de su existencia por yacimientos como La Bastida (Martínez Santa-Olalla et al. 1947; García del Toro 1983; Buikstra et al. 1990; Ayala 1991; Martínez Rodríguez 1995; Martínez Rodríguez y Ponce 2000a, 2000b). Se trata de urnas cerámicas depositadas bajo los niveles de habitación a modo de enterramiento, incluso con ajuares en algunos casos. Sin embargo, ninguna contenía restos humanos $\mathrm{y}$, por ello, se han interpretado como elementos de posibles rituales conmemorativos (Martínez Rodríguez et al. 1996).

\section{2b. Los ajuares argáricos}

Llegamos así al punto clave valorado por la investigación: los ajuares funerarios. Ciertamente, en muchas tumbas argáricas, los vivos depositaron determinados objetos junto a sus muertos. No obstante, esta práctica afectó sólo a un número específico de enterramientos argáricos. De las 167 tumbas analizadas aquí, 104 contenían ajuar $(63 \%)$ y 63 no $(38 \%)$.

"Ajuar funerario" es un término genérico que engloba aspectos y prácticas de diferente tipo. Por regla general, incluye todos aquellos elementos que acompañaban al difunto o a la difunta dentro de su propio espacio o en un pequeño receptáculo adyacente que forma parte de la propia tumba (como ocurre en algunas necrópolis, fundamentalmente murcianas y almerienses). Responde a diferentes realidades: desde elementos ligados a prácticas cotidianas a otros que podrían haber tenido una funcionalidad mucho más restringida, como por ejemplo, la ritual. Parece plausible que, en algunos casos, se depositaran objetos manufacturados en vida por los propios difuntos, como nos lo indica la aparición de pe- 
queños recipientes cerámicos de manufactura tosca en las tumbas de algunos niños (Sánchez Romero 2008a).

Tradicionalmente, se ha considerado que los ajuares funerarios argáricos estaban integrados por ornamentos de piedra, hueso o metal -plata y oro inclusive-, recipientes cerámicos -manufacturados en muchos casos con una finalidad únicamente ritual-, instrumentos óseos o metálicos, como punzones y agujas, y las consideradas armas -básicamente espadas y alabardas metálicas-. Las hachas y los cuchillos/puñales, también metálicos, han cabalgado entre una u otra de las dos últimas denominaciones. Unas pocas categorías se han asociado con uno u otro sexo (los punzones y diademas con las mujeres y las espadas, alabardas y hachas con los hombres), pero la mayoría de los objetos se encuentran tanto en las tumbas de las mujeres como en las de los hombres.

Sobre todo a partir de los años 80 , se utilizó el estudio de estos ajuares funerarios para proponer claras diferencias sociales en el seno de las comunidades argáricas y un acceso desigual a los recursos económicos y al poder ideológico-político. Así, Lull y Estévez (1986), tras examinar 396 sepulturas individuales, concluyeron que en El Argar habrían convivido cinco categorías de enterramientos con una lectura claramente social. Junto a este análisis general para todo el grupo argárico otros estudios trataron de establecer diferencias sociales a partir de los ajuares de necrópolis específicas. Así en la Cuesta del Negro los resultados del análisis estadístico de los ajuares mostrarían la existencia de 4 categorías asimiladas a grupos sociales (Molina 1983; Contreras et al. 1987-88). Además, en otros poblados como el Cerro de la Encina (Aranda y Molina 2006) o Fuente Álamo (Schubart et al. 2000) se han podido establecer áreas espacialmente diferenciadas en función del tipo de ajuar encontrado en sus tumbas.

Recientemente, además, han comenzado a plantearse novedosas interpretaciones basadas en los rituales de comensalidad asociados al ritual funerario argárico. Evidencias de estas prácticas serían la producción de un conjunto normalizado de vasijas cerámicas asociadas con la presentación y consumo de alimentos y bebidas. Sus destacadas propiedades visuales estarían relacionadas con prácticas sociales de exhibición y escenificación. De igual forma, la aparición de restos faunísticos en las sepulturas argáricas permite plantear el sacrificio de bóvidos y ovicápridos, principalmente, para su consumo compartido en rituales funerarios. Una porción de carne de una de las extremidades de estas especies animales formaría parte del ajuar funerario. La adscripción social de los difuntos/as determinaría el tipo de carne consumida. Así, los bóvidos serían sacrificados sólo en las ceremonias de los sectores sociales más elevados, mientras que en el ritual dedicado a los niños y niñas menores de 12 años y a las personas de un nivel social inferior se consumirían ovejas y/o cabras (Aranda y Esquivel 2006, 2007; Sánchez Romero et al. 2007; Aranda y Montón-Subías e.p.).

\section{MUERTE, CUERPOS E IDENTIDAD FEMENINA EN EL ARGAR}

\section{3a. El comportamiento funerario con las mujeres}

Hace ya cierto tiempo que la disciplina arqueológica indaga sobre la identidad social y el simbolismo asociado a la cultura material de los enterramientos del pasado (Hodder 1984; Morris 1988; O’Shea 1996; Scarre 1994; Parker Pearson 1999; Gillespie 2001; Chesson 2001; Joyce 2001; Arnold y Wicker 2001). Por mi parte, estoy convencida de que el estudio del comportamiento funerario puede ilustrarnos sobre los diferentes mecanismos que se utilizaron para construir la identidad en las sociedades del pasado. Las comunidades argáricas trataron a sus difuntos muy posiblemente de acuerdo con la percepción social que tenían de ellos. Dicha percepción, a su vez, vendría condicionada por las actividades desempeñadas en vida y por el contexto de interacción en el que se llevaran a cabo, hecho que a su vez contribuiría a la formación de un tipo de identidad o identidades específicas.

El carácter intencional de la cultura material depositada en las tumbas argáricas resulta evidente: se eligen unos elementos y no otros. Proceden de un amplio abanico de prácticas: desde las cotidianas, relacionadas en algunos casos con las actividades de mantenimiento y la gestión de la vida diaria, hasta las más excepcionales por su naturaleza específicamente ritual. 
Anteriormente, se ha señalado que, a partir del estudio de los ajuares funerarios, se han identificado diferentes clases sociales en el mundo argárico. Para ello, se ha privilegiado la discontinuidad material, es decir, lo que diferencia a unos grupos de otros. Además, estos grupos (y las personas que los integran) se han considerado como entidades monolíticas, unitarias e impermeables, definidas en oposición. Sin embargo, asumiendo que las personas no son entidades sociales aisladas sino que interactúan en contextos relacionales abiertos y dinámicos, es posible pensar en adscripciones sociales más complejas que las tradicionalmente supuestas (Montón-Subías 2007).

Ya hace tiempo que el análisis constructivista de la identidad social viene enfatizando la pluralidad de la adscripción social (Jenkins 1997). Igualmente, la investigación feminista, tanto desde fuera de la arqueología (Rosaldo y Lamphere 1974; Lerner 1993; Phoenix y Pattynama 2006) como desde dentro (Gero y Scattolini 2002; Brumfiel 2006; Montón-Subías 2007), ha insistido en el hecho de que los grupos sociales no son unitarios si se considera la dimensión de género, que a su vez posee diferentes matices. Creo que eso ocurre también en las sociedades argáricas, donde los mecanismos relacionales con que se contruye la identidad femenina son transversales a los diferentes grupos sociales que pueden establecerse para estas poblaciones. Posteriormente lo analizaré a partir de los procesos de inclusión y exclusión que pueden deducirse del estudio de la cultura material con la que se entierran las mujeres.

Disciplinas como la psicología, la antropología, la filosofía o las ciencias de la comunicación han enfatizado que, a pesar de que la identidad humana se forma a partir de una serie de mecanismos diversos, dos son los más importantes (Geertz 1973; Markus y Kitayama 1998). En el primer caso, el de la llamada identidad relacional, ésta se desarrolla a partir de las relaciones sociales con los otros siendo la vinculación con el resto del grupo el núcleo de la identidad; en el segundo caso, la identidad, denominada individualizada, se construye a medida que el individuo se desvincula de los demás y prima aquellas experiencias y características que le son propias (Min-Sun 2001). Desde la antropología se ha señalado que ambas identidades pueden coexistir (LiPuma 2001) y, desde la arqueología,
Almudena Hernando (2002, 2003) ha intentado desentrañar los mecanismos identitarios a través de los cuales puede explicarse dicha coexistencia.

Para el caso concreto de la cultura de El Argar, cabe preguntarse sobre los elementos transversales que aparecen en todas las tumbas femeninas expresivos de la combinación entre la identidad relacional e individualizada. Se sabe, sin embargo, que salvo el enterramiento en sí no existe ningún otro aspecto común a todas las sepulturas argáricas. Desde mi punto de vista, uno de los aspectos más interesantes del ritual funerario argárico reside precisamente en que la coiné funeraria reconocible en las diferentes áreas de la geografía argárica se manifiesta de forma muy diferente. Por ello, como apuntaba previamente, el ritual funerario argárico vincula y desvincula, une y separa, iguala y diferencia a las distintas personas que vivieron en estas comunidades. La cultura material funeraria forma parte también de los mecanismos mediante los que se representan y construyen elementos relacionales e individualizadores en la creación social de las identidades argáricas. Desde esta perspectiva el estudio del ritual funerario argárico abre nuevas vías para la interpretación de estas sociedades.

Como, al margen del enterramiento, nada iguala en el mundo funerario al conjunto de las mujeres argáricas, la diferencia más obvia es la que establece entre quienes se entierran con y sin ajuar. En la muestra analizada la presencia de ajuares en enterramientos femeninos es altamente significativa. Tan solo un enterramiento individual no contiene ajuar (la T56 de Fuente Álamo) y, respecto a los dobles, tan solo la T26 y T36 de Gatas y la T16 del Cerro de la Encina. Las tumbas triples y cuádruples con mujeres presentan siempre ajuar. Es decir, que de las 52 tumbas que son de o contienen mujeres, solo cuatro carecen de ajuar.

Veamos pues qué elementos diferenciaron y conectaron a estas mujeres. Ya los Siret apuntaron que el uso de diademas aparecía sólo en las tumbas de mujeres y, el de punzones, principalmente en ellas (Siret y Siret 1890). Más tarde, Lull y Estévez (1986) demostraron la significación estadística de la anterior observación apuntando además que el punzón se asociaba en muchas ocasiones al puñal/cuchillo, que había una preferencia por la combinación del punzón con 
adornos y una tendencia a presentar plata. Por su parte, los hombres se caracterizarían por la presencia exclusiva de alabardas, hachas y espadas, por la aparición significativa de cerámicas de formas bicónicas y/o puñales y por la preferencia en la presencia de oro.

\section{3b. Ofrendas exclusivas y ajuares femeninos}

Desafortunadamente, todas las diademas conocidas en el mundo argárico proceden de excavaciones antiguas. Ya los Siret indicaron que algunas de las tumbas que las contenían se hallaban muy deterioradas y que parte de los ajuares podían incluso haberse perdido (Siret y Siret 1890: 194-195). Sólo se conocen nueve diademas en todo el mundo argárico: ocho de plata (en El Argar, Gatas, El Oficio y Fuente Álamo) y una de oro (en Caravaca de la Cruz). No se trata, por lo tanto, de un fenómeno generalizado, sino de una peculiaridad básicamente restringida a muy pocas tumbas dentro de su zona nuclear (Lull 1983). La diadema marcaría una doble o triple exclusividad, si se confirma su restricción geográfica: de género, de posición social, al encontrarse en las tumbas femeninas con mayor amortización de elementos funerarios, y geográfica. Mediante la posesión de este elemento ornamental, un reducidísimo grupo de mujeres se diferenciaría del resto. Los enterramientos masculinos carecen de elementos exclusivos ornamentales. Las ofrendas paralelizables, por su carácter exclusivo, lo constituyen las espadas y las alabardas, que a veces proceden de tumbas dobles con mujeres con diadema. De todos modos, contamos con un mayor número de espadas y alabardas que, además, se distribuyen por toda la geografía argárica.

Un caso extremadamente diferente es el del punzón, por razones diversas: se distribuye por toda la geografía argárica, puede hallarse en cualquier tumba que contenga ajuar, está vinculado a las prácticas cotidianas de la comunidad y aparece con más frecuencia que otros elementos metálicos funerarios en contextos no funerarios (Fig. 2).

El hallazgo de punzones en unas pocas tumbas masculinas impide asociarlos exclusivamente a las mujeres, aunque sí se puede afirmar que la vinculación punzón-mujer es altamente significativa desde un punto de vista estadístico. Esa asociación se ha destacado en repetidas ocasiones
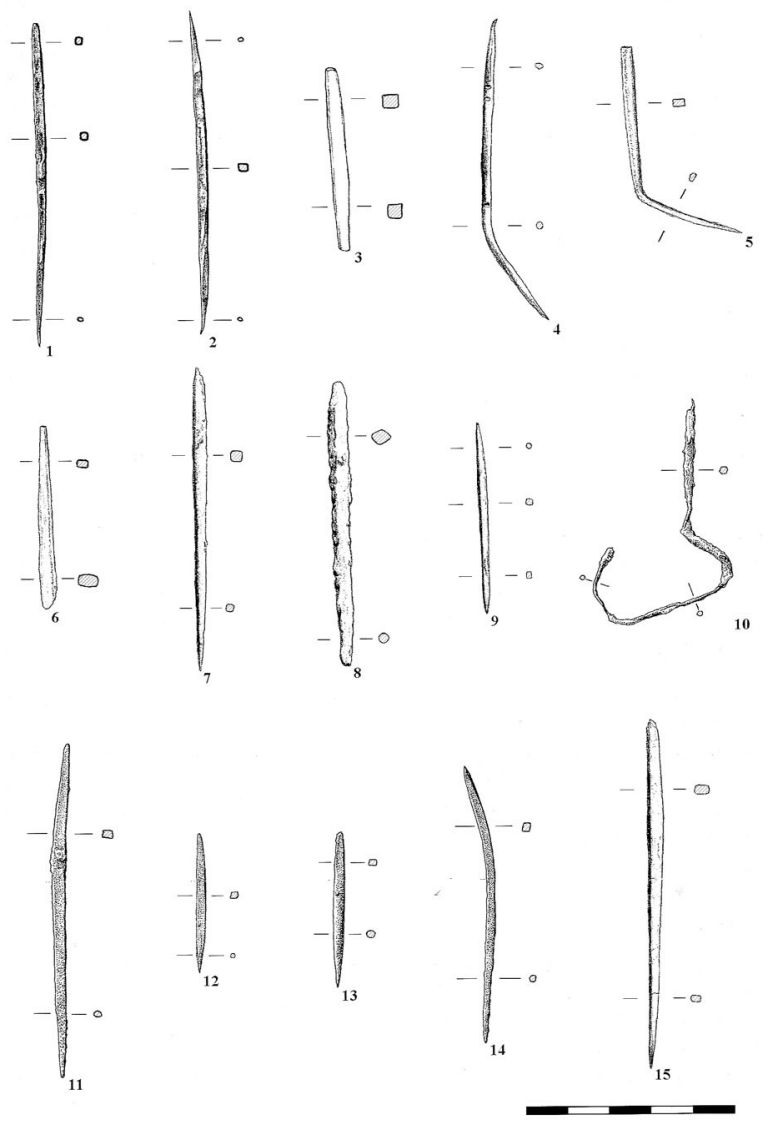

Fig. 2. Punzones argáricos (según Contreras 2000).

(Siret y Siret 1890; Lull y Estévez 1986; Castro et al. 1993-4; Pingel et al. 2003), aunque sólo recientemente se ha empezado a discutir su papel en la representación y construcción de la identidad femenina (Montón-Subías 2007; Aranda et al. 2009b). Destaco cinco aspectos fundamentales en relación a este utensilio:

1. Su transversalidad social. Potencialmente, puede aparecer en cualquier tumba con ajuar, desde aquéllas con mayor número de objetos funerarios amortizados, como muchas de las tumbas con diademas (por ejemplo, la T454, T62 y T38 de El Argar; la T2 de Gatas y la T6 de El Oficio), hasta las que únicamente contienen un punzón (Fig. 3).

2. Su transversalidad temporal. Es el único elemento metálico asociado significativamente a un sexo durante toda la cultura argárica. Por el contrario, la aparición y perduración de los elementos metálicos exclusivos varía más en las tumbas masculinas. 


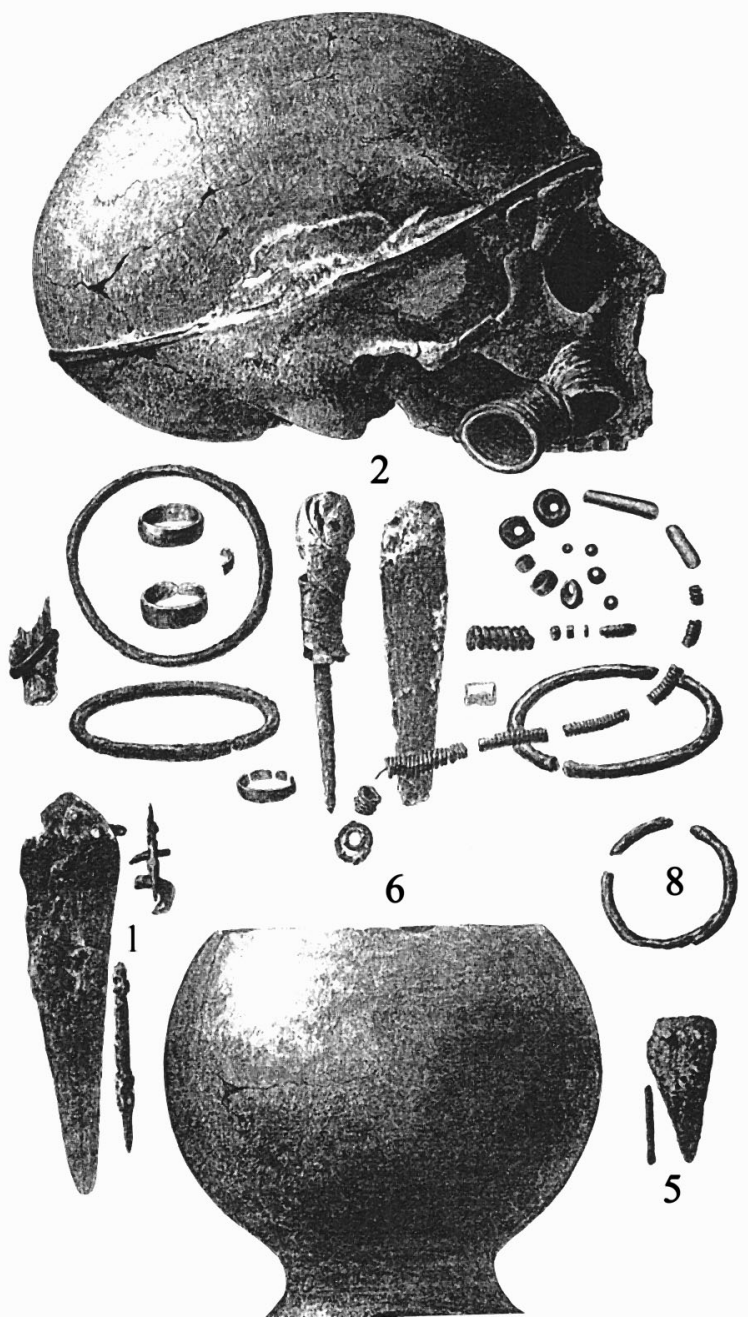

Fig. 3. Diferentes tumbas del yacimiento argárico de Gatas (Turre, Almería) (según Siret y Siret 1890). Puede apreciarse que los punzones aparecen en tumbas con grandes diferencias en la deposición de objetos funerarios.

3. El amplio rango de edad de las muertas con quienes se entierran. Con tan solo una excepción para todo el mundo argárico (Lull et al. 2004), los elementos metálicos exclusivamente asociados a los hombres no se encuentran en tumbas infantiles. De todos modos, debo advertir que se desconoce el sexo de los esqueletos infantiles con punzones. En la muestra estudiada dos individuos infantiles poseen punzón: la posible niña de entre 9 y 10 años de la T101 de Fuente Álamo, y el individuo infantil de 6 años de la T5 de Peñalosa. Es posible que algunos de los otros individuos indeterminados hallados en Castellón Alto, el Cerro de San Cristóbal y El Rincón de Almendricos sean también infantiles, pero se carece de información respecto a su edad. Sí se puede atestiguar que el punzón se vincula a personas cuya edad oscila entre los 6 y los 50-70 años. Al haberse demostrado la tendencia estadísticamente significativa de su asociación con mujeres adultas, algunos investigadores han propuesto que las tumbas infantiles en que aparecen sean de niñas (Castro et al. 1993-4; Lull et al. 2004). Lo mismo se ha planteado para sexuar las tumbas infantiles en las que aparecen elementos exclusivos de mujeres en otros lugares arqueológicos, sean punzones (Weglian 2001) o agujas (Rega 2000). Sin embargo, no se puede descartar la posibilidad de que los punzones se hallen tanto en tumbas de niños como de niñas, ya que podría suceder que ambos participasen en las tareas que realizaran sus madres, como sugiere Hamlin (2001).

4. Su aparición en contextos no funerarios. El punzón es el elemento metálico asociado significativamente a un sexo que aparece con mayor frecuencia en dichos contextos. Tal vez el caso mejor estudiado sea el de Peñalosa, donde, en las excavaciones modernas, se han contabilizado hasta 13 punzones (dos de ellos incompletos) en contextos no funerarios frente a cuatro en contextos funerarios (Moreno 2000). Normalmente, se les ha englobado en un grupo uniforme (Montero 1994: 61), aunque también se han sugerido diferencias morfológicas entre los punzones funerarios y no funerarios, pues Moreno (2000) señala que los primeros son de mayor tamaño. Sólo el punzón en plata de la tumba 7 de Fuente Álamo no podría servir como instrumento de trabajo (Siret y Siret, 1890), pero tampoco en este caso puede discernirse si se trata de un objeto personal o de un elemento manufacturado especialmente para ser enterrado con la muerta.

5. Su probable vinculación con las denominadas actividades de mantenimiento, vinculadas a la gestión diaria de la cotidianeidad.

Dichas actividades corresponden a prácticas cotidianas recurrentes, que regulan y estabilizan el ciclo de la vida diaria en una comunidad y posibilitan su continuidad en el tiempo. Relacionadas sobre todo con el cuidado y bienestar de los miembros del grupo, incluyen, además del propio cuidado, las actividades culinarias, textiles, higiénicas y sanitarias, la socialización de niños y niñas, y la organización y acondicionamiento de los espacios relacionados (Picazo 1997; Colomer 
et al. 1998; Montón-Subías y Sánchez-Romero 2008).

Siguiendo a Hamlin (2001: 125), parece plausible que cuando existe un vínculo tan estrecho entre un instrumento y un sexo es porque ese sexo lo empleó en sus actividades. Desafortunadamente, no se han realizado aún análisis sobre el uso dado a los punzones argáricos, aunque paralelos etnográficos y textuales nos los conectan con una serie de labores cotidianas relacionadas con el trabajo de la piel y la madera, la manufactura textil, la cestería, el mantenimiento y reparación de ciertos objetos, etc. (Spector 1993).

Ante lo dicho, llama la atención que la aparición de estos utensilios en el registro funerario cruce todas las circunstancias sociales menos el sexo, por lo que es posible que sea precisamente aquí donde radique el sentido de su recurrente vinculación con las mujeres.

Partiendo de un trabajo anterior (Aranda et al. $2009 \mathrm{~b}$ ), he vuelto a evaluar estadísticamente la asociación punzón-mujer, incluyendo más utensilios que los recogidos entonces. A los yacimientos anteriormente analizados, Gatas y Fuente Álamo (en Almería), Peñalosa (en Jaén), Cerro de la Encina y Fuente Amarga (en Granada) y Lorca -Calle Zapatería 11, Convento de las Madres Mercedarias y Los Tintes-, Los Cipreses y El Bajil (en Murcia) (Schubart y Arteaga 1986; Schubart et al. 1987, 1993, 2000; Eiroa 1993-94, 2004; Castro et al. 1995; Martínez Rodríguez et al. 1996; 1999; Fresneda et al. 1999; Contreras et al. 2000; Kunter 2000; Martínez Rodríguez y Ponce 2002a, 2002b; Aranda y Molina 2006), he añadido los de Castellón Alto, Cuesta del Negro, Cerro de San Cristóbal y Punete de Noy (Granada), el Culantrillo, el Rincón de Almendricos y Santa Catalina del Monte (Murcia) y Illeta dels Banyets y El Tabayà (Alicante) (García 1963; Molina y Pareja 1975; Molina et al. 2004; Fresneda et al. 1991; Molina Fajardo 1983; García 1963; García del Toro y Ayala 1978; García del Toro 1983; Ruiz 1988; Llobregat 1986; Hernández 1990).

Se cuenta ahora con un total de 167 tumbas y 231 esqueletos: 54 pertenecen a mujeres (si se incluyen tres que se presentan como probables); 53 a hombres y, en 124 casos, no se ha podido determinar el sexo, debido a un estado de conservación deficiente, a la ausencia de partes diagnósticas, a que este dato no se ha publicado $\mathrm{y}$, principalmente, a que la mayoría corresponden a enterramientos infantiles (92). De las mujeres, 21 se enterraron solas (contando las 3 probables); 25 en tumbas dobles; 6 en tumbas triples y 2 en cuádruples.

Entre los ajuares funerarios había 37 punzones (35 de cobre y 2 de hueso). Dos de ellos se encontraron en tumbas cuádruples $\mathrm{y}$, afortunadamente, uno se pudo asociar al cuerpo de la mujer durante el proceso de excavación. Tres punzones más se encontraron en tumbas triples, pudiéndose relacionar uno con el cuerpo de una mujer. De los trece punzones encontrados en tumbas dobles, solo dos se pudieron vincular con mujeres. Diez se encontraron en tumbas individuales femeninas; tres en tumbas individuales masculinas y dos en tumbas infantiles. Para el resto, no se dispone de información sobre la edad y el sexo del esqueleto. En total, por lo tanto, 14 punzones pertenecen con seguridad a mujeres, 3 a hombres y dos a niños o niñas.

Se realizaron dos pruebas de chi-cuadrado. En la primera se consideró que, además de los punzones asociados a tumbas individuales femeninas, todos los que se encontraban en tumbas dobles y tumbas infantiles pertenecían a mujeres y/o niñas. El resultado arrojó un valor de $\mathrm{p}=0.0001$. En la segunda prueba, tan sólo se incluyeron como punzones femeninos los que con seguridad pertenecían a mujeres y se excluyeron las tumbas infantiles. El valor obtenido también fue de $\mathrm{p}=$ 0.0001. Ello indica que la diferencia observada en la presencia de punzones entre hombres y mujeres es estadísticamente muy significativa y que la explicación no se debe al azar.

Además de los punzones y las diademas, no se ha identificado ningún otro ítem o elemento ritual que pueda asociarse significativamente a las tumbas de mujeres.

Es factible preguntarse ahora sobre los mecanismos que generaron la construcción de la identidad femenina argárica. No obstante, resumiré primero la información que nos proporciona el análisis de los cuerpos argáricos.

\section{3c. Los cuerpos del pasado}

Al estudiar los mecanismos mediante los cuales se construyó la identidad femenina en las sociedades argáricas, partiendo de la hipótesis de que la identidad humana se configura en un contexto de prácticas de interacción social, no podría 
ignorarse la información clave sobre esas prácticas que aporta el estudio de los cuerpos enterrados y las posibles diferencias existentes entre hombres y mujeres. El cuerpo no es un simple objeto que deba ser estudiado en relación a la cultura, sino que debe ser entendido como sujeto de cultura, o en otras palabras, como el ámbito existencial de la cultura. El concepto de embodiment ha sido adaptado a la antropología, la etnografía y a la arqueología como una forma de tratar el cuerpo como auténtico campo para la cultura, ya que la existencia de los seres humanos no es separable del cuerpo con que se experimenta la vida (Sánchez Romero 2008b: 8).

Según Roberts (1995), el desarrollo de un cuerpo humano no está únicamente relacionado ni con los procesos biológicos que rigen su crecimiento y envejecimiento ni con la herencia genética. The human body is plastic and the specific way in which a body develops is related to the totality of the environment in which a person is situated, where environment is understood as comprising both so-called natural and cultural phenomena, including the actions of self and others in the world. (Roberts 1995, cit. en Sofaer 2006: 160). Dicha plasticidad hace que el cuerpo humano sea receptivo a diferentes influencias, entre las que se encuentran las actividades físicas, que también pueden dejar huellas sobre su esqueleto.

Las colecciones de restos humanos argáricos han sido estudiadas por diferentes equipos de antropólogos a lo largo de más de un siglo. No obstante ha sido en las dos últimas décadas cuando se han desarrollado importantes análisis que han permitido caracterizar pormenorizadamente a estas poblaciones (Botella 1975; Botella et al. 1986, 1995; Buikstra et al. 1990; Kunter 2000, 2004; Jiménez-Brobeil et al. 1995, 2004, 2007; Ortega et al. 1995; Contreras et al. 2000; De Miguel 2001; Al-oumaqui et al. 2004; Aranda et al. 2008).

Debido a las múltiples causas que pueden dejar su rastro en los esqueletos y para obtener una imagen más precisa de las diferentes actividades que practicaron hombres y mujeres en las sociedades argáricas, el estudio que aquí se resumirá combinó los resultados proporcionados por dos indicadores fundamentales: la artrosis y el estrés músculo-esquelético (Jiménez-Brobeil y Ortega 1992; Jiménez-Brobeil et al. 1995, 2004).
La artrosis es una enfermedad crónica y degenerativa que afecta al hueso cuando los cartílagos se desgastan y degeneran. El factor más determinante en su aparición parece ser la edad, siendo la menopausia un momento crítico para las mujeres, pero también inciden en su formación la genética, el metabolismo y las actividades físicas.

$\mathrm{Su}$ incidencia en las poblaciones argáricas se ha evaluado a partir de una muestra de 110 individuos procedentes fundamentalmente de las necrópolis granadinas de Castellón Alto y Cuesta del Negro (69 \%) y, en menor medida, de la necrópolis de Fuente Amarga, Cerro de la Encina, Cerro de la Virgen y Terrera del Reloj (Jiménez-Brobeil y Ortega 1992; Jiménez-Brobeil et al. 1995, 2004). Los restos humanos analizados pertenecen a 53 varones y 57 mujeres que se han distribuido en las categorías de adultos (21-40 años), maduros (41-60 años) y seniles (mas de 60 años).

Hay más hombres que mujeres afectadas por esta patología: $38 \%$ y $25,9 \%$ respectivamente, auque las diferencias no son estadísticamente significativas (Jiménez-Brobeil et al. 1995). El estudio de las articulaciones y conjuntos articulares anatómicos sí permite establecerlas. Se han analizado de forma individualizada las articulaciones del hombro, codo, muñeca, cadera, rodilla y tobillo y los conjuntos articulares de la columna lumbar, dorsal y cervical (Fig. 4). En la columna vertebral las diferencias entre ambos sexos no son significativas a excepción del sector dorsal. En el miembro superior los varones muestran una afec-

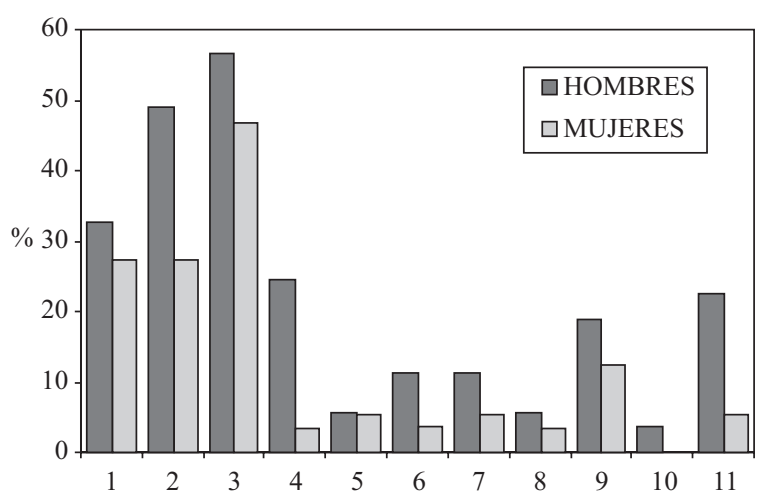

Fig. 4. Representación gráfica de los niveles artrósicos entre hombres y mujeres. 1: Columna Cervical; 2: Columna Dorsal; 3: Columna Lumbar; 4: Hombro; 5: Muñeca; 6: Mano; 7: Cadera; 8: Rodilla; 9: Tobillo; 10: Pie. (según Jiménez-Brobeil et al. 2004). 
tación del hombro muy acusada ya desde la edad adulta, mientras que en las restantes articulaciones, sobre todo en el codo, las diferencias entre sexos no son muy destacadas. En el miembro inferior, los valores de los varones tampoco exceden de forma considerable a los de las mujeres, con la excepción del conjunto del pie, donde, de nuevo, la diferencia resulta estadísticamente significativa (Jiménez-Brobeil et al. 1995, 2004).

El segundo marcador de actividad, el estrés músculo-esquelético, observa los cambios en las inserciones de ligamentos y tendones debidos a un incremento en el desarrollo muscular. De la muestra de 110 individuos se analizaron los marcadores de estrés, eliminando a los individuos de más de 60 años para evitar la influencia de la edad en el desarrollo muscular. Se seleccionaron 15 marcadores para reflejar las principales articulaciones del cuerpo y se indicó la incidencia del estrés músculo-esquelético en cada una de ellas (Fig. 5). Los resultados demostraron que los hombres experimentaban un estrés músculo-esquelético mayor y que este debía explicarse

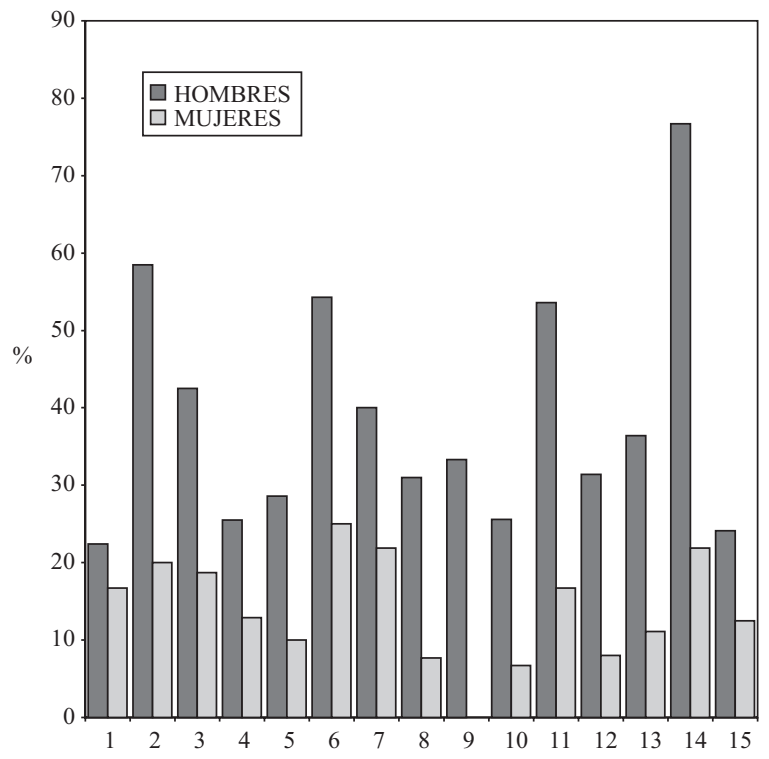

Fig. 5. Representación gráfica de la frecuencia de marcadores de estrés músculo-esquelético por individuos y sexos. 1: Escápula; 2: Pectoral mayor; 3: Redondo mayor; 4: Deltoides; 5: Olécranon; 6: Supinador; 7: Radio; 8: Trocánter mayor; 9: Trocánter menor; 10: Línea áspera; 11: Rótula; 12: Línea poplítea; 13: Tuberosidad tibial; 14: Tendón de Aquiles; 15: Espolón (según Jiménez-Brobeil et al. 2004). por razones diferentes al dimorfismo sexual (Al-oumaqui et al. 2004; Jiménez-Brobeil et al. 2004).

Los hombres presentaban un fuerte desarrollo del miembro superior respecto a las mujeres, con una fuerte incidencia en los marcadores de los músculos pectoral mayor, supinador corto y redondo mayor. El primero rota el húmero hacia dentro y eleva el tronco aproximándolo al brazo; el segundo permite la rotación del antebrazo de adentro hacia fuera, y el tercero facilita la elevación del hombro y aductor del brazo. En los tres casos las diferencias son altamente significativas respecto a las mujeres, que presentan valores medianos en los marcadores del miembro superior, claramente inferiores a los de la serie masculina. Dentro del grupo de las mujeres, el mayor desarrollo se centra en los músculos que doblan el antebrazo sobre el brazo (Jiménez-Brobeil et al. 2004).

Los hombres exhiben frecuencias medianas o altas en los marcadores del miembro inferior. El mayor estrés se produce en los músculos que extienden el tobillo y la rodilla o que extienden la pierna sobre el muslo, todos ellos relacionados con la acción de andar. Dentro de las frecuencias halladas destaca la cifra relativamente alta del marcador del espolón, cuyo desarrollo se ha relacionado con un sobreesfuerzo al caminar por terrenos abruptos. Las mujeres presentan frecuencias bajas o muy bajas de los marcadores del miembro inferior. Los músculos relacionados con la actividad de caminar también tienen los valores más altos. Ello sugiere que ambos sexos realizaron una actividad similar con intensidades muy diferentes (Jiménez-Brobeil et al. 2004). Estas importantes diferencias supondrían la práctica de actividades diferenciadas entre hombres y mujeres basadas en patrones de movilidad muy acusados en el caso de los hombres y mucho más limitados en las mujeres.

La consideración conjunta de los resultados de ambos índices (artrosis y estrés músculo-esquelético) pareció apuntar hacia una diferencia clara en los patrones de actividad masculinos y femeninos. Se constató, además, que la artrosis también afectaba a aquellas zonas del cuerpo con mayor estrés músculo-esquelético: las articulaciones de las vértebras dorsales, de los hombros y de los pies. Por ello, la artrosis pudo estar relacionada, al menos en algunas articulaciones, con actividades físicas intensas. Esta relación resultó aún más 
relevante al coincidir estos índices en los mismos esqueletos (Contreras et al. 2000; Miguel 2001; López- Padilla et al. 2006; Aranda et al. 2008).

Los análisis paleoantropológicos sugieren un nivel de movilidad distinto, más reducido para las mujeres, hecho que concuerda con la realización de las actividades de mantenimiento, más ligadas a los espacios incluidos en el propio poblado.

\section{LA IDENTIDAD FEMENINA EN EL ARGAR: CONCLUSIONES}

Probablemente, en las comunidades argáricas se combinaron elementos individualizadores y relacionales en la construcción de la identidad personal. No obstante, pienso que los elementos relacionales tuvieron mucho mayor peso en la configuración de la identidad femenina. Como he afirmado previamente, la identidad se construye en un contexto de interacción social donde las prácticas y acciones cotidianas adquieren una importancia fundamental ya que confieren a quienes las realizan una manera determinada de relacionarse con los otros, estar en el mundo, entenderlo y experimentarlo.

Tanto los resultados de los análisis paleoantropológicos como algunos elementos simbólicos depositados en las tumbas de mujeres, vinculan a éstas con la práctica de las actividades de mantenimiento. Quienes las desempeñan suelen construir su identidad personal a partir de la relación con el resto de la comunidad. Creo que este tipo de identidad relacional se expresa simbólicamente mediante la introducción del punzón en muchas tumbas femeninas argáricas.

Afirmaba anteriormente que la aparición de estos utensilios en el registro funerario atraviesa todas las circunstancias sociales menos el sexo y que, por eso, su significado simbólico probablemente deba buscarse ahí. Ya en artículos anteriores avancé la hipótesis de que dicha identidad relacional presentaría también un carácter transversal (Montón-Subías, 2007) y destaqué el hecho de que la cultura material simbólica del mundo de la muerte recogiese también las actividades de mantenimiento (Montón-Subías 2007; Aranda et al. 2009b). Esto se constata en muchas otras sociedades prehistóricas, aunque su significado simbólico haya pasado desapercibido en muchas ocasiones. Precisamente, lo que deseo enfatizar es que este tipo de cultura material vinculada con las actividades de mantenimiento formó parte también de las estrategias complejas y el universo simbólico que se expresaron en los rituales funerarios y constituyó uno de los mecanismos mediante los que expresar y recrear una identidad relacional y transversal.

La cultura material integrada en las prácticas de mantenimiento cotidianas operaría a modo de significante simbólico de la identidad femenina en el ritual funerario. Parece lógico, además, que los punzones sean los que con más frecuencia aparecen en contextos habitacionales no funerarios. La posibilidad de que algunos punzones funerarios pudiesen haberse manufacturado específicamente con fines rituales (como algunos de Peñalosa o el de la T7 de Fuente Álamo) podría enfatizar la interpretación de estos punzones como elementos o atributos de identidad.

Los punzones se convertirían en un nexo de unión que denotaría continuidad en varios ámbitos: continuidad cronológica durante todo el período argárico, continuidad a lo largo y ancho de la escala social y continuidad entre el contexto cotidiano de la vida y de la muerte.

Por descontado, ello no implica necesariamente que todas las mujeres argáricas, ni siquiera todas las que se enterraron con punzones, llevasen a cabo todas estas tareas. Tampoco estoy afirmando que las mujeres argáricas integrasen un conglomerado social monolítico. Debieron existir adscripciones sociales diversas, como se desprende de las diferencias en la cantidad y calidad de los ajuares funerarios femeninos o en el material sobre el que se manufacturan los punzones: hueso, cobre e incluso plata, que lo hace inservible para su uso práctico, lo que acentúa su carácter simbólico. Desconozco, además, si cada mujer podría marcar atributos individuales en elementos accesorios a los punzones, como, por ejemplo, los enmangues o sus fundas o en otras piezas ornamentales como los collares. Sí sé que unas pocas mujeres se diferenciaron del resto por el uso de elementos exclusivos (diademas) e incluso por su manera de enterrarse: mientras que la mayoría de las mujeres se entierran en decúbito lateral, la mujer adulta de la T12 del Convento de las Madres Mercedarias lo hace en decúbito supino, con las piernas enfrentadas. No obstante, muchas de estas mujeres presentan también punzones entre sus ajuares funerarios, incluida la enterrada en 
Madres Mercedarias, lo que refuerza el carácter transversal de los mismos.

Propongo que el punzón, en tanto que elemento integrado en las prácticas relacionadas con el mantenimiento cotidiano de la comunidad, probablemente a cargo de muchas mujeres argáricas, pudiera haberse elegido para representar y enfatizar simbólicamente en el registro funerario la identidad femenina predominante, aunque en algunos casos este simbolismo solo se hubiese tomado prestado en el momento de la muerte. Este simbolismo identificaría y recrearía la percepción y concepción que en las comunidades argáricas se tuvo de las mujeres. Esto no implicaría, como afirman, entre otros, Markus y Kitayama (1991) o La Fontaine (1985), que todas aquellas personas caracterizadas por una concepción interdependiente del propio ser, incluidas las mujeres argáricas, careciesen de particularidades propias (en forma de rasgos internos, características o preferencias), sino que no serían el elemento decisivo que guiaba su comportamiento social en el seno de sus comunidades.

\section{AGRADECIMIENTOS}

Deseo expresar mi más sincero agradecimiento a Gonzalo Aranda Jiménez y Margarita Sánchez Romero, a quienes considero coautores de este artículo por su generosa colaboración en todos los procesos que condujeron a su presentación. Vaya también mi agradecimiento para los evaluadores/as anónimos/as, cuyos comentarios mejoraron sin duda el manuscrito final entregado.

\section{BIBLIOGRAFÍA}

Alfaro, G. 1984: Tejido y cestería en la Península Ibérica. Historia de su técnica e industrias desde la prehistoria hasta la romanización. Bibliotheca Praehistorica Hispana XXI, CSIC. Madrid.

Al-Oumaqui, I.; Jiménez-Brobeil, S.A. y Souich, P.D. 2004: "Markers of activity patterns in some populations of the Iberian Peninsula". International Journal of Osteoarchaeology 14: 343-359.

Andúgar, L. 2006: "La colección argárica del Museu d'Arqueologia de Catalunya". Cypsela 16: 227-242.
Aranda, G. y Esquivel, J.A. 2006: "Ritual funerario y comensalidad en las sociedades de la Edad del Bronce del Sureste Peninsular: la Cultura de El Argar". Trabajos de Prehistoria 63 (2): 117-133.

Aranda, G. y Esquivel, J.A. 2007: "Poder y prestigio en las sociedades de la cultura de El Argar. El consumo comunal de bóvidos y ovicápridos en los rituales de enterramiento". Trabajos de Prehistoria 64 (2): 95-118.

Aranda, G. y Molina, F. 2006: "Wealth and Power in the Bronze Age of South-east of Iberian Peninsula: the Funerary Record of Cerro de la Encina". $O x$ ford Journal of Archaeology 25 (1): 47-59.

Aranda, G.; Molina, F.; Fernández, S.; Sánchez Romero, M.; Al Oumaqui, A.; Jiménez-Brobeil, S. y Roca, M.G. 2008: "El poblado y necrópolis argáricos del Cerro de la Encina (Monachil, Granada). Las campañas de excavación de 2003-2005". Cuadernos de Prehistoria y Arqueología de la Universidad de Granada 18: 219-264.

Aranda, G. y Montón-Subías, S. e.p.: "Feasting Death. Funerary rituals in the Bronze Age societies of south-eastern Iberia”. En G. Aranda, S. Montón-Subías y M. Sánchez-Romero (eds.): Guess who is coming to dinner. Commensality rituals in the prehistoric societies of Europe and the Near East. Oxbow. Oxford.

Aranda, G.; Montón-Subías, S. y Jiménez-Brobeil, S. 2009a: "Conflicting Evidence? Weapons and Skeletons in the Bronze Age of southeast Iberia". Antiquity 83 (322): 1038-1051.

Aranda, G.; Montón-Subías, S.; Sánchez-Romero, M. y Alarcón, E. 2009b: "Death and everyday life: the Argaric societies from Southeast Iberia". Journal of Social Archaeology 9 (2): 139-162.

Arnold, B. y Wicker, N. 2001: Gender and the Archaeology of Death. Altamira Press. Walnut Creek (CA).

Arteaga, O. 1992: "Tribalización, jerarquización y Estado en el territorio de El Argar". Spal 1: 197-208.

Ayala, M.M. 1991: El poblamiento argárico en Lorca. Estado de la cuestión. Real Academia Alfonso X el Sabio. Murcia.

Binford, L. 1971: "Mortuary practices: their study and their potential". En J. Brown (ed.): Approaches to the Social Dimensions of Mortuary Practices. Memoir of the Society for American Archaeology. Washington: 390-420.

Blance, B. 1964: "The Argaric Bronze Age in Iberia". Revista Guimaraes LXXIV: 192-142.

Blance, B. 1971: Die Anfänge der Metallurgie auf der Iberischen Halbinsel. Studien zu den Anfängen der Metallurgie. Berlín.

Botella, M. 1975: "Paleopatología en la necrópolis argárica de la Cuesta del Negro, Purullena (Grana- 
da)". Actas del IV Congreso Español de Historia de la Medicina (Granada 1973): 21-28. Granada.

Botella, M.; Escoriza, T. y Cabañete, M.J. 1986: "La mortalidad en las poblaciones argáricas". Homenaje a Luis Siret (1934-1984). Consejería de Cultura de la Junta de Andalucía. Sevilla: 467-472.

Botella, M.; Jiménez-Brobeil, S. y Ortega, J.A. 1995: "Traumatisms in Bronze Age settlements in the Iberian Peninsula: Argar Culture". Proceedings of the IXth European Meeting of the Paleopathology Association (Barcelona 1992): 65-72. Barcelona.

Brandherm, D. 2000: "El poblamiento argárico de Las Herrerías (Cuevas de Almanzora, Almería), según la documentación inédita de L. Siret". Trabajos de Prehistoria 57 (1): 157-172.

Brown, J.A. (ed.) 1971: Approaches to the Social Dimensions of Mortuary Practices. Memoir of the Society for American Archaeology 25. Washington.

Brumfiel, E. 2006: "Methods in Feminist and Gender Archaeology: A feeling for difference-likeness". En S. Nelson (ed.): Handbook of Gender in Archaeology. Altamira Press. Lanham: 31-58.

Buikstra. J.; Castro, P.; Chapman, R.; González-Marcén, P.; Hoshower, L.; Lull, V.; Picazo, M.; Risch, R. y Sanahuja, M.E. 1990: "La necrópolis de Gatas". Anuario de Arqueología Andaluza II: 261-276.

Cámara, J.A. 2001: El ritual funerario en la Prehistoria Reciente en el Sur de la Península Ibérica. British Archaeological Repports. International Series 913. Oxford.

Castro, P.V.; Chapman, R.; Gili, S.; Lull, V.; Micó, R.; Rihuete, C. y Sanahuja, M.E. 1993-94: "Tiempos sociales de los contextos funerarios argáricos". Anales de Prehistoria y Arqueología 9-10: 77-106.

Castro, P.V.; Lull, V.; Micó, R. y Rihuete, C. 1995: “La prehistoria reciente en el sudeste de la Península Ibérica. Dimensión socio-económica de las prácticas funerarias". En R. Fábregas, F. Pérez y C. Fernández (eds.): Arqueoloxía da Morte na Península Ibérica desde as Orixes ata o Medievo. Excmo. Concello de Xinxo de Limia. Xinxo de Limia: 127-167.

Castro, P.V.; Chapman, R.; Gili, S.; Lull, V.; Micó, R.; Rihuete, C.; Risch, R. y Sanahuja, M. E. 2001: "La sociedad argárica". En M. Ruiz-Gálvez (ed.): La Edad del Bronce, ¿Primera Edad de Oro de Espana? Crítica. Barcelona: 118-216.

Chapman, R. 1990: Emerging complexity: the later prehistory of Southeast Spain, Iberia and the West Mediterranean. Cambridge University Press. Cambridge.

Chapman, R. 2003: Archaeologies of complexity. Routledge. Londres.
Chapman, R. 2005: "Mortuary Analysis. A Matter of Time?". En J.E. Buikstra, L.A. Beck, y S.R. Williams (eds.): Interacting with the Dead. Perspectives on Mortuary Archaeology for the New Millenium. University Press of Florida. Gainsville: 25-40.

Chapman, R.; Kinnes, I. y Randsborg, K. (eds.) 1981: The Archaeology of Death. Cambridge University Press. Cambridge.

Chesson, M. 2001: "Social memory, Identity and Death: An Introduction”. En M. Chesson (ed.): Social memory, Identity, and Death: Anthropological Perspectives on Mortuary Rituals. American Anthropological Association. Arlington: 1-10.

Childe, V.G. 1945: "Direccional changes in funerary practices during 50,000 years". Man 4: 13-19.

Colomer, L; González Marcén, P. y Montón-Subías, S. 1998: "Maintenance Activities, Technological Knowledge and Consumption Patterns: a View from a Prehistoric Iberian Site (Can Roqueta, 1200-500 cal BC)". Journal of Mediterranean Archaeology 11:53-80.

Contreras, F. (ed.) 2000: Proyecto Peñalosa. Análisis histórico de las comunidades de la Edad del Bronce del piedemonte meridional de sierra Morena y depresión Linares-Bailén. Consejería de Cultura. Junta de Andalucía. Sevilla.

Contreras, F.; Capel, J.; Esquivel, J.A.; Molina, F. y la Torre, F. de 1987-88: "Los ajuares cerámicos de la necrópolis argárica de la Cuesta del Negro (Purullena, Granada). Avance al estudio analítico y estadístico". Cuadernos de Prehistoria de la Universidad de Granada 12-13: 135-156.

Cuadrado, E. 1950: "Útiles y armas de El Argar. Ensayo de tipología". Actas del I Congreso Nacional de Arqueología (Almería 1949): 7-28. Cartagena.

Damm, C. 1991: "From Burials to Gender Roles: Problems and Potentials in Post-Processual Archaeology". En D. Walde y N. Willows (eds.): The Archaeology of Gender. Proceedings of the Twenty-Second Annual Conference of the Archaeological Association of the University of Calgary. University of Calgary Archaeological Association. Calgary: 130-135.

Delibes, G. 2000: “Cinabrio, huesos pintados en rojo y tumbas de ocre: ¿prácticas de embalsamamiento en la Prehistoria?". En M. Olcina y J.A. Soler Díaz (eds.): Scripta in Honorem Enrique Llobregat Conesa. Instituto Alicantino de Cultura Juan Gil-Albert. Alicante: 223-236.

Díaz-Andreu, M.; Lucy, S.; Babic, S. y David, N. 2005: The archaeology of Identity. Approaches to gender, age, status, ethnicity and religion. Routledge. Londres y Nueva York.

Eiroa, J. 1993-94: "Aspectos funerarios del poblado de El Bajil (Moratalla, Murcia). (Niveles de la Edad 
del Bronce)". Anales de Prehistoria y Arqueología 9-10: 55-76.

Eiroa, J. 2004: La Edad del Bronce en Murcia. Real Academia Alfonso X el Sabio. Murcia.

Fowler, C. 2004: The Archaeology of Personhood. An anthropological approach. Routledge. Londres y Nueva York.

Fresneda, E.; Rodríguez Ariza, O.; López, M y Peña, J.M. 1991: "Excavación de urgencia en el Cerro de San Cristóbal (Ogíjares, Granada). Campañas de 1988 y 1989”. Anuario Arqueológico de Andalucía III: 233-239.

Fresneda, E.; Rodríguez-Ariza, O.; López, M. y Peña, J.M. 1999: "El asentamiento argárico de Fuente Amarga". Actas del XXIV Congreso Nacional de Arqueología (Cartagena 1997): 231-240. Murcia.

García, M. 1963: "El poblado argárico del Cerro el Culantrillo en Gorafe (Granada)". Archivo de Prehistoria Levantina X: 69-96.

García del Toro, J. 1983: "La necrópolis argárica de El Rincón de Almendricos (Lorca, Murcia)". Actas del XVI Congreso Nacional de Arqueología (Cartagena 1982): 217-222.

García del Toro, J. y Ayala, M.M. 1978: "La necrópolis argárica 'El Rincón' en Almendricos (Lorca)”. Murcia 14.

Geertz, C. 1973: The Interpretation of Culture: Selected Essays. Basic Books. Nueva York.

Gero, J. y Scattolini, M.C. 2002: "Beyond Complementarity and Hierachy: New definitions for archaeological gender relations". En S. Nelson y M. Rosen-Ayalon (eds.): In pursuit of gender: worldwide archaeological approaches. Altamira Press. Walnut Creek: 155-171.

Gillespie, S. 2001: "Mortuary Ritual, Agency and Personhood. A Case Study from the Ancient Maya". Journal of Anthropological Archaeology 20 (1): 73-112.

Gilman, A. 1981: "The development of social stratification in Bronze Age Europe". Current Anthropology 22: 1-23.

Gilman, A. 2001: "Assessing political development in Copper and Bronze Age southeast Spain". En J. Haas (ed.): From leaders to rulers. Kluwer Academic/Plenum. Nueva York: 59-81.

Hamlin, C. 2001: "Sharing the Load: Gender and Task Division at the Windover Site". En B. Arnold y N. Wicker (eds.): Gender and the Archaeology of Death. Altamira Press. Walnut Creek: 119-35.

Hernández, M. 1990: "Un enterramiento argárico en Alicante". Homenaje a Jerónimo Molina. Accademia Alfonso X El Sabio. Murcia: 87-94.

Hernando, A. 2002: Arqueología de la Identidad. Akal. Madrid.

Hernando, A. 2003: "En la Prehistoria no vivieron 'individuos'. Sobre los problemas de aplicación de la
Teoría de la Acción a las sociedades prehistóricas". Era-Arqueología 6: 84-99.

Hodder, I. 1984: "Burials, houses, women and men in the European Neolithic". En D. Miller y C. Tilley (eds.): Ideology, Power and Prehistory. Cambridge University Press. Cambridge: 51-68.

Insoll, T. 2007: The Archaeology of Gender Identities. A Reader. Routledge. Londres y Nueva York.

Jenkins, R. 1997: Rethinking ethnicity: Arguments and Explorations. Sage. Londres.

Jiménez-Brobeil, S.A. y Ortega, J.A. 1992: “Osteoartritis de la columna vertebral en las poblaciones de la Edad del Bronce en la provincia de Granada". Munibe 8: 257-260.

Jiménez-Brobeil, S.; Botella, M. y Ortega, J.A. 1995: "Arthropaties in the Iberian Peninsula during the Bronze Age: Argar culture". Proceedings of the IXth European Meeting of the Paleopathology Association (Barcelona 1992): 173-179. Barcelona.

Jiménez-Brobeil, S. A.; Al-Oumaqui, I. y Esquivel, J.A. 2004: "Actividad física según el sexo en la cultura argárica. Una aproximación desde los restos humanos". Trabajos de Prehistoria 62 (2): 141-154.

Jiménez-Brobeil, S.A.; Al-Oumaqui, I. y Souich, P.D. 2007: "Childhood Trauma in Several Populations from the Iberian Peninsula". International Journal of Osteoarchaeology 17:189-198.

Jover, F.J. y López Padilla, J. 1995: “El Argar y el Bronce valenciano. Reflexiones en torno al mundo funerario". Trabajos de Prehistoria 52 (1): 71-86.

Jover, F.J. y López Padilla, J. 1997: Arqueología de la Muerte. Las prácticas funerarias en los límites de El Argar. Universidad de Alicante. Alicante.

Joyce, R. 2001: "Burying the Dead at Tlatilco: Social memory and Social identities". En M. Chesson (ed.): Social memory, Identity, and Death: Anthropological Perspectives on Mortuary Rituals. American Anthropological Association. Arlington: 12-26.

Kunter, M. 2000: "Los Restos de esqueletos humanos hallados en Fuente Álamo durante las campañas de 1985, 1988 y 1991". En H. Schubart, V. Pingel y O. Arteaga (eds.): Fuente Álamo. Las excavaciones arqueológicas 1977-1991 en el poblado de la Edad del Bronce. Consejería de Cultura de la Junta de Andalucía. Sevilla: 265-782.

Kunter, M. 2004: "Die Menschilichen Skekettreste aus den grabungskampagnen 1996 und 1999 in Fuente Álamo". Madrider Mitteilungen 45: 88-96.

La Fontaine, J. 1985: "Person and Individual: some anthropological reflections". En M. Carrithers, S. Collins y S. Lukes (eds.): The Category of the person. Cambridge University Press. Cambridge: 123-140. 
Lerner, G. 1993: "Reconceptualizing Differences Among Women". En A. Jagger y P.S. Rothenberg (eds.): Feminist Frameworks: Alternative Theoretical Accounts of the Relation Between Women and Men. McGraw Hill. Nueva York: 237-248.

Li Puma, E. 2001: Encompassing Others. The Magic of Modernity in Melanesia. The University of Michigan Press. Ann Arbor.

López Padilla, J.; Belmonte, D. y Miguel, M.P. de 2006: "Los enterramientos argáricos de la Illeta dels Banyets de El Campello. Prácticas funerarias en la frontera oriental de El Argar". En J.A. Soler (ed.): La ocupación prehistórica de la Illeta dels Banyets (El Campello, Alicante). Diputación de Alicante. Alicante: 118-171.

Lull, V. 1983: La cultura de El Argar. Un modelo para el estudio de las formaciones sociales prehistóricas. Akal. Madrid.

Lull, V. 2000: "Argaric society: death at home". Antiquity 74: 581-590.

Lull, V. y Estévez, J. 1986: “Propuesta metodológica para el estudio de las necrópolis argáricas". Homenaje a Luis Siret (1934-1984). Consejería de Cultura de la Junta de Andalucía. Sevilla: 441-442.

Lull, V. y Picazo, M. 1989: “Arqueología de la muerte y estructura social". Archivo Español de Arqueología 62: 5-20.

Lull, V.; Rihuete, C.; Micó, R. y Risch, R. 2004: “Las relaciones de propiedad en la sociedad argárica. Una aproximación a través del análisis de las tumbas de individuos infantiles". Mainake XXVI: 233-272.

Lull, V. y Risch, R. 1996: “El estado argárico”. Verdolay 7: 97-109.

Llobregat, E. 1986: "Illeta dels Baneyts". Arqueología en Alicante 1976-1986: 63-67.

Markus, H. y Kitayama, S. 1998: "The cultural psychology of personality". Journal of Cross-Cultural Psychology 29: 63-87.

Martínez Rodríguez, A. 1995: "I fase de excavaciones de urgencia en la calle Zapatería n. ${ }^{\circ} 11$ (Lorca)". Memorias de Arqueología 1987-1988: 64-80.

Martínez Rodríguez, A.; Ponce, J. y Ayala, M.M. 1996: Las prácticas funerarias de la Cultura Argárica en Lorca (Murcia). Caja de Ahorros de Murcia y Excmo. Ayuntamiento de Lorca. Murcia.

Martínez Rodríguez, A.; Ponce, J. y Ayala, M.M. 1999: “Excavaciones de urgencia del poblado argárico de Los Cipreses, Lorca. Años 1992-93”. Memorias de Arqueología 8: 156-182.

Martínez Rodríguez, A. y Ponce J. 2002a: "Excavación arqueológica de urgencia en el subsuelo de la antigua iglesia del convento de las Madres Mercedarias (C/ Zapatería-C/ La Cava, Lorca)". Memorias de Arqueología 10: 90-137.

Martínez Rodríguez, A. y Ponce J. 2002b: "Segunda intervención arqueológica en la Plaza Juan Moreno n. ${ }^{\circ}$, confluencia con la calle Los Tintes, (Lorca)". Memorias de Arqueología 10: 150-160.

Martínez Santa-Olalla, J.; Sáez Martín, B; Posac Mon, C.; Sopranos Alto, J.A.; Val Caturla, E. 1947: Excavaciones en la ciudad del Bronce Mediterráneo II, de la Bastida de Totana (Murcia). Informes y Memorias 16. Comisaría General de Excavaciones Arqueológicas. Madrid.

Meskell, L. 2001: "Archaeologies of Identity". En I. Hodder (ed.): Archaeological Theory Today. Polity Press. Cambridge: 187-213.

Miguel, M.P. de 2001: "Inhumaciones argáricas de la Illeta dels Banyets (El Campello, Alicante): aproximación paleopatológica". En J.A. Sánchez (ed.): $V$ Congreso Nacional de Paleopatología (Alcalá la Real 1999): 9-19. Alcalá la Real.

Min-Sun, K. 2001: "Perspectives on Human Communication. Implications for Transculture Theory". En V. Milhouse, M. Asante y P. Nwosu (eds.): Transcultural Realities. Interdisciplinary Perspectives on Cross-Cultural Relations. Sage. Londres: 3-31.

Molina Fajardo, F. 1983: “Almuñécar en el marco de la cultura Argárica”. En F. Molina Fajardo (ed.): Almuñécar. Arqueología e Historia. Caja Provincial de Ahorros Granada. Granada: 3-20.

Molina, F. y Pareja, E. 1975: Excavaciones en la Cuesta del Negro (Purullena, Granada). Campaña de 1971. Excavaciones arqueológicas en España 86. Madrid.

Molina, F.; Rodríguez-Ariza, M.O.; Jiménez, S. y Botella, M. 2003: "La sepultura 121 del yacimiento argárico de El Castellón Alto (Galera, Granada)". Trabajos de Prehistoria 60 (1): 153-158.

Molina, F.; Rodríguez-Ariza, M.O.; Haro, M.; Alfonso, J.A. y Navas, E. 2004: “Actuaciones arqueológicas en el yacimiento de Castellón Alto (Galera, Granada). Campaña de 2001". Anuario Arqueológico de Andalucía III: 435-443.

Montero, I. 1994: El origen de la metalurgia en el Sudeste de la Península Ibérica. Instituto de Estudios Almerienses. Almería.

Montón-Subías, S. 2007: "Interpreting archaeological continuities: an approach to transversal equality in the Argaric Bronze Age of South-east Iberia". World Archaeology 39 (2): 256-62.

Montón-Subías, S. y Sánchez-Romero, M. (eds.) 2008: Engendering Social Dynamics: The Archaeology of Maintenance Activities. British Archaeological Reports, International Series 1862. Archaeopress, Oxford.

Moreno, A. 2000: "La metalurgia de Peñalosa". En F. Contreras (ed.): Proyecto Peñalosa: Análisis histórico de las Comunidades de la Edad del Bronce del Piedemonte Meridional de Sierra Morena y Depresión Linares-Bailén. Junta de Andalucía, Consejería de Cultura. Granada: 167-221. 
Morris, I. 1988: Burial in ancient society. Cambridge University Press. Cambridge.

O'Shea, J. 1996: Villagers of the Maros: A Portrait of an Early Bronze Age Society. Plenum Press. Nueva York.

Ortega, J.A.; Jiménez-Brobeil, S.A. y Botella, M. 1995: "Paleopathology in the Terrera del Reloj necropolis, Dehesas de Guadix, Granada (Spain)". En R. Batista, D. Campillo y T. Carreras (eds.): IXth European Meeting of the Paleopathology Association (Barcelona 1992): 241-48. Barcelona.

Parker Pearson, M. 1999: The archaeology of Death and Burial. Sutton Publishing. Stroud.

Peebles, C. y Kus, S. 1977: "Some archaeological correlates of ranked societies". American Antiquity 42: 421-448.

Phoenix, A. y Pattynama, P. 2006: "Intersectionality". European Journal of Women's Studies 13: 187-192.

Picazo, M. 1997: "Hearth and Home: The Timing of Maintenance Activities". En J. Moore y S. Scott (eds.): Invisible People and Processes: Writing Gender and Childhood into European Archaeology. Leicester University Press. Leicester y Nueva York: 59-67.

Pingel, V.; Schubart, H.; Arteaga, O.; Roos, A.M. y Kunst, M. 2003: "Excavaciones arqueológicas en la ladera sur de Fuente Álamo. Campaña de 1999”. Spal 12: 179-230.

Randsborg, K. 1974: "Social stratification in Early Bronze Age Denmark". Prähistorische Zeitschrift 49: 38-61.

Rautman, A.E. y Talalay, L.E. 2000: "Introduction. Diverse Approaches to the Study of Gender in Archaeology". En A.E. Rautman (ed.): Reading the Body. Representations and Remains in the Archaeological Record. University of Pennsylvania Press. Philadelphia: 1-12.

Rega, E. 2000: "The gendering of children in the Early Bronze Age cemetery at Mokrin”. En M. Donald y L. Hurcombe (eds.): Gender and Material Culture in Historical Perspective. Macmillan. Londres: 238-249.

Roberts, D. 1995: "The pervasiveness of plasticity". En M. Taylor y B. Bogin (eds.): Human Variability and Plasticity. Cambridge University Press. Cambridge: 1-17.

Rosaldo, M.Z. y Lamphere. L. 1974: Women, Culture and Society. Standford University Press. Standford.

Ruiz, M.J. 1988: "Excavaciones de urgencia en el poblado de Sta. Catalina del Monte (Verdolay, Murcia)". Memorias de Arqueología 7: 78-116.

Sánchez Romero, M. 2008a: "An approach to learning and socialization in children during the Spanish Bronze Age". En L.H. Dommasnes y M. Wrigglesworth (eds.): Children Identity and the past. Cambridge Scholars Publishing. Cambridge: 113-124.

Sánchez Romero, M. 2008b: "Cuerpos de mujeres: la construcción de la identidad y sus manifestaciones durante la Edad del Bronce". Arenal 15 (2): 5-29.

Sánchez Romero, M.; Aranda, G. y Alarcón, E. 2007: "Gender and Age Identities in Rituals of Commensality. The Argaric Societies”. En P. González-Marcén, C. Masvidal, S. Montón-Subías y M. Picazo (eds.): Interpreting household practices: reflections on the social and cultural roles of maintenance activities. Treballs d'Arqueologia 13: $69-89$

Scarre, C. 1994: "The meaning of death: funerary beliefs and the prehistorian". En C. Renfrew y E. Zubrow (ed.): The ancient mind. Elements of cognitive archaeology. Cambridge University Press. Cambridge: $75-82$.

Schubart, H. 1975: “Cronología relativa de la cerámica sepulcral de El Argar". Trabajos de Prehistoria 32: $78-92$.

Schubart, H. y Arteaga, O. 1986: "Fundamentos Arqueológicos para el estudio socio-económico y cultural del área de el Argar”. Homenaje a Luis Siret (1934-1984). Consejería de Cultura de la Junta de Andalucía. Sevilla: 289-307.

Schubart, H.; Arteaga, O. y Pingel, V. 1987: “Fuente Álamo (Almería): informe preliminar sobre la excavación de 1985 en el poblado de la Edad del Bronce”. Anuario Arqueológico de Andalucía 1985 (II): 305-312.

Schubart, H.; Pingel, V. y Arteaga, O. 1993: “Informe preliminar sobre la excavación realizada en 1991 en el poblado de la Edad del Bronce. Fuente Álamo". Anuario Arqueológico de Andalucía 1991 (II): 24-27.

Schubart, H.; Pingel, V. y Arteaga, O. 2000: Fuente Álamo. Las excavaciones arqueológicas 19771991 en el poblado de la Edad del Bronce. Arqueología Monográficas 9, Junta de Andalucía, Consejería de Cultura. Sevilla.

Shanks, M. y Tilley, C. 1982: "Ideology, symbolic power and ritual communication: a reinterpretation of Neolithic mortuary practices". En I. Hodder (ed.): Symbolic and Structural Archaeology. Cambridge University Press. Cambridge: 129-54.

Shennan, S.E. 1975: "The social organisation at Branc". Antiquity 49: 279-87.

Siret, E. y Siret, L. 1890: Las primeras edades del metal en el sudeste de España. Barcelona.

Sofaer, J. 2006: "Gender, Bioarchaeology and Human Ontogeny". En R. Gowland y C. Knüsel (eds.): Social Archaeology of funerary remains. Oxbow Books. Oxford: 155-167. 
Spector, J. 1993: What this awl means? Feminist Archaeology at a Wahpeton Dakota Village. Minnesota Historical Society Press. St. Paul, Minnesota.

Tarlow, S. 1999: Bereavement and Commemoration. An Archaeology of Mortality. Blackwell. Oxford.
Weglian, E. 2001: "Grave Goods do not a Gender make. A Case Study from Singen and Hohenwiel, Germany". En B. Arnold and N. Wicker (eds.): Gender and the Archaeology of Death. Altamira Press. Walnut Creek (CA): 137-155. 